\title{
Impact of Cloud Cover on Global UV Irradiance over Baghdad, Iraq
}

\author{
Kais J. AL-Jumaily, Natiq A. Zaki, Ali M. AL-Salihi \\ Department of Atmospheric Sciences College of Science, Al-Mustansiriyah University Baghdad, Iraq
}

\begin{abstract}
Six months of continuous of hourly records of Ultraviolet irradiance recorded at Baghdad, (33.34 ${ }^{o} \mathrm{~N}, 44.45^{\circ} \mathrm{E}$, $34 \mathrm{~m}$ a.m.s.l.), Iraq, were combined with concurrent synoptic cloud observations to investigate the relative influence of clouds on UV irradiance. The ratio of UV irradiance to the total solar irradiance increases with cloud cover, especially for cloud cover greater than 4 octas, In order to determine the effect of cloud on UV irradiance, a cloud modification factor was considered, it is defined as the ratio between the UV measurements and the corresponding clear sky UV irradiance that would be expected for the same time period and atmospheric conditions. It was shown that the effect of cloud with 4 octas increases demonstrating the spectral dependence of the cloud radiative extinction, while the impact of cloud cover less than 4 octas is relatively constant and ranged between $(3.6-4.3 \%)$.
\end{abstract}

Keywords : Ultraviolet Radiation, Clouds, Ozone, Baghdad, Iraq.

\section{INTRODUCTION}

The study of solar ultraviolet radiation has received considerable attention in the past few years because of its biological, ecological, and physic effects produced by short-wave radiation received at the surface of the earth. Solar UV radiation is usually divided into three bands UV-C (100-280 nm) which is completely absorbed by stratospheric ozone; UV-B $(280-315 \mathrm{~nm})$ which is only partially absorbed or scattered in the atmosphere and UV-A (315-400 $\mathrm{nm}$ ) which makes up most of the UV radiation received at the earth's surface[1-8]. In outer space, UV-B and UV-A band represent only 7.45\% of the total solar radiation [9]. The stratospheric ozone forms a shield around the globe protecting the biosphere from the dangerous ultraviolet radiation coming from the sun. A slight decrease in the stratospheric ozone leads to an increase in the UV-B reaching the earth. UV radiation data are of particular interest because such radiation is energetic enough to break apart several biological molecules, including DNA [4]. The increase in UV radiation affects human health [10]. There are wide spatial and temporal variations in the UV irradiance at the surface of the earth depending on latitude, solar elevation, and atmospheric and local conditions. Atmospheric aerosols also affect the UV irradiance, but their influence is small relative to cloud cover [11-13]. Among these factors influencing the UV irradiance, cloud cover present a high temporal and spatial variability. It is certain that clouds can cause large year to year variability in UV radiation and therefore possibility play an important role in determining long term trends [14]. For the cloud effect, it is necessary to have knowledge of clouds optical thickness and drop size distributions. The aim of this work is to investigate the effects of clouds on UV irradiance using data collected in Baghdad, Iraq.

\section{LOCATION OF STUDY AND MEASUREMENTS}

Broadband solar irradiance $(0.3-3 \mu \mathrm{m})$, global, ultraviolet global irradiance, photo synthetically active irradiance $(0.4-0.7 \mu \mathrm{m})$ and other meteorological variables were continuously recorded for the period between first of October 1994 and 30 March 1995 in the Radiometric Station of the Center of Energy and Environment Researches $\left(33.34{ }^{0} \mathrm{~N}, 44.45{ }^{0} \mathrm{E}, 34 \mathrm{~m}\right.$ a.m.s.l.), in Baghdad, Iraq. The measurements were taken every 10 minutes. Solar global irradiance was measured using Eppely pyranometer, An Eppley TUVR radiometer was used to measure the ultraviolet irradiance $(0.29-0.385 \mu \mathrm{m})$ on a horizontal surface. Air temperature and relative humidity were also recorded for the same period. From this database, hourly values have been generated for the mentioned period to guarantee that a wide range of seasonal conditions and solar elevation angles are covered. It is important to point out that there are significant differences in temporal and cosine response between the radiometers used for the measurements of UV and broadband solar irradiance. Consequently hourly values have been computed, in order to reduce the temporal effects that could be introduced in obtaining the ratio of UV radiation to broadband solar radiation. These undesirable effects can reach quite a significant level, especially under highly changing conditions, which are usually associated with broken cloud fields.

\section{RESULTS AND DISCUSSION}

To study the effect of cloud on UV irradiance, a cloud modification factor, which is define as the ratio between the UV measurement and corresponding clear sky UV irradiance that would be calculated for the same 
time period and atmospheric condition, was considered. In this work a model, which tested in Al-Salihi [1], was used to account for the UV irradiance under clear sky conditions. Thus, this model allows estimation of cloudless UV irradiance. The UV irradiance data were classified according to cloud cover observations. Additionally, the data were classified into several categories according to the sun elevation. Four categories have been considered and were characterized by sun elevation angles, $\theta\left(60^{\circ}<\theta 1,40^{\circ}<\theta 2<60^{\circ}, 20^{\circ}<\theta 3<40^{\circ}\right.$, and $10^{\circ}<\theta 4<20^{\circ}$ ). For each sun elevation and cloud cover categories, the mean and standard deviation of UV irradiance were computed. Figure (1). Presents the dependence of UV irradiance on cloudiness degrees for the different sun elevation.

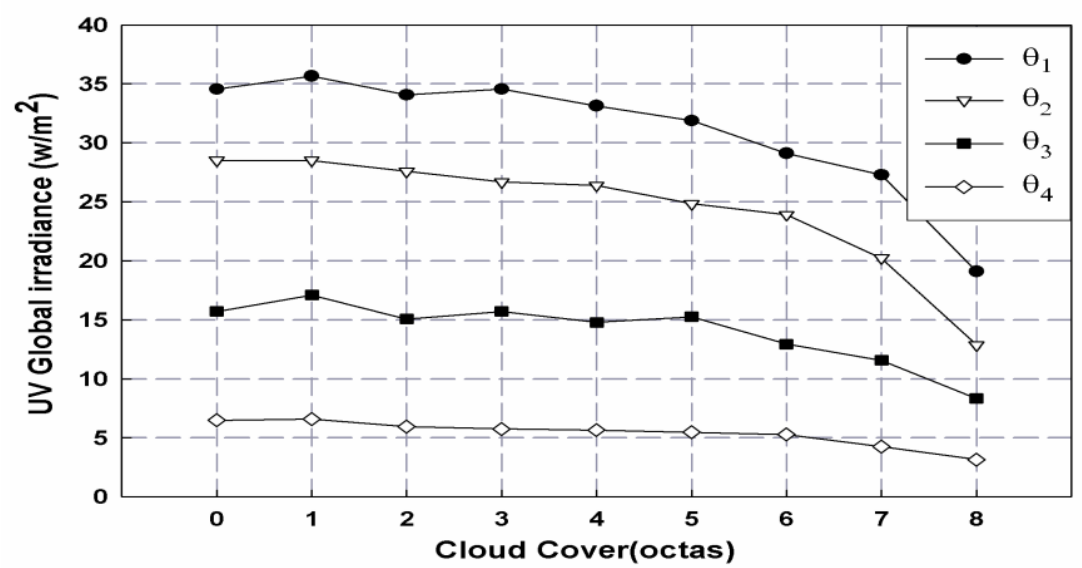

Figure1.Ultraviolet global irradiance vs. cloud cover for different solar elevation angles

The effect of cloud is clearly evident for high cloud cover but negligible for fractional cloud cover below 3 octas suggesting that, for these cloud covers, clouds tend to be located out of the line between the sun and sensor, it also can be noticed in Fig (1) that the high solar elevations and overcast skies yields relatively similar results of associated effects for low solar elevation and cloudless skies. The performance of UV irradiance under cloudy conditions is similar to that followed by the solar irradiance in other spectral [13], It could be useful to analyze the performance of a dimensionless ratio like that obtained as the ratio of UV irradiance to the total solar radiation, G. total solar irradiance. The ratio on UV/G provides insight into the relative importance of UV irradiance of total solar irradiance at the surface. This ratio has been studied by different authors [14 - 16], Figure (2) shows the dependence of UV/G on cloud cover for the same sun elevation angel categories considered in the previous analysis of UV irradiance.

A first evident from figure (2) is that the ratio UV/G increases with cloud cover, and this increase is more evident for cloud cover greater than 4 octas, This means that there are some differences in the effect of cloud on total solar irradiance and UV irradiance. This increase demonstrates the spectral dependence of the cloud radiative extinction.

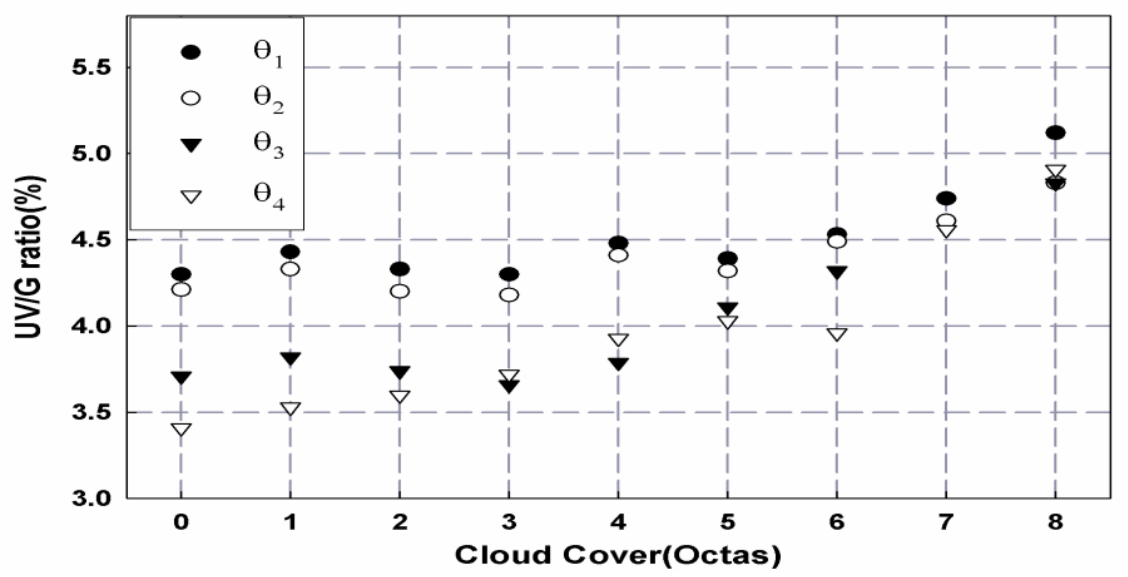

Figure 2. Ratio of global ultraviolet irradiance to total solar irradiance UV/G vs cloud cover for different solar elevation angles 
In this sense, a greater absorption in the near infrared region than in shorter wavelength causes an enhancement of the UV range relative to the total solar spectrum [17]. On other hand, although the differences are rather small, it seems that for higher solar elevations UV/G presents greater values than for lower solar elevations, at least for cloudless and partially cloudless conditions. The increase of UV/G with cloud cover is greater for lower solar elevations and thus for over cast conditions the mean UV/G value does not depend on the solar elevation analysis.

To study this relationship furthermore the index $\mathrm{K}_{\text {tuv }}$ was analyzed, this index is defined as the ratio between the UV irradiance reaching the surface level and the corresponding extraterrestrial flux $U_{\text {ext }}[18]$, that is:

$$
K_{t u v}=\frac{U V}{U V_{\text {ext }}}
$$

Where : $\mathrm{UV}_{\mathrm{ext}}$ is the $\mathrm{UV}$ extraterrestrial solar radiation on a horizontal surface, it is given by:

$$
U V_{\text {ext }}=\frac{I_{\text {scuv }} \operatorname{Sinj\alpha }}{L}
$$

And $\alpha$ is the solar elevation and $\mathrm{L}$ is the sun-earth distance in astronomical units.

The solar constant for UV, $\mathrm{I}_{\text {SCUV }}$, has been taken as $78 \mathrm{w} / \mathrm{m}^{2}$ from spectral values given by Lenbole, [19].

Figure (3) shows the dependence of $\mathrm{UV} / \mathrm{G}$ ratio and $\mathrm{K}_{\mathrm{tuv}}$ with cloud coverage for data recorded around noon and demonstrates the opposite trends of this ratio as function of cloudless conditions, the $\mathrm{K}_{\mathrm{tuv}}$ index had a value of about 0.45 and the UV/G ratio was close to $4 \%$. The different trends for this ratio: as the cloud cover increased lead to an overcast value of about $5 \%$ for $\mathrm{UV} / \mathrm{G}$ while the reduced value of $\mathrm{K}_{\mathrm{tuv}}$ index was close to 0.2.

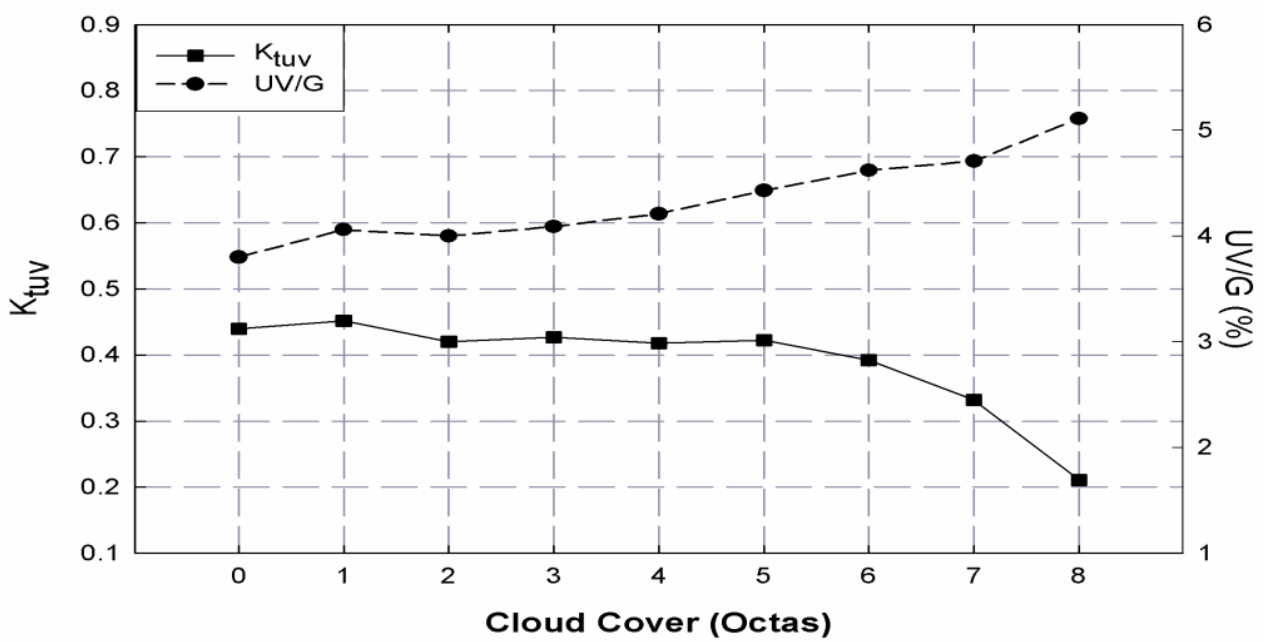

Figure 3. Ratio of global ultraviolet irradiance to total solar irradiance UV/G and ultraviolet transmittance $\mathrm{K}_{\mathrm{tuv}}$, vs cloud cover .

Since the global irradiance (Ultraviolet or solar total radiation) varies widely under different sky conditions, it's convenient to normalize the global irradiance to minimize variability and maximize the generality of the results, for this reason, the performance of UV global irradiance was analyzed and normalized to the value under cloudless sky.

$$
F_{U V}=\frac{U V}{U V_{o}}
$$

$\mathrm{UV}_{\mathrm{o}}$ can be estimated by application of any simple radiative transfer model such as (SMARTS 2.9.5) [20]. where $\mathrm{UV}$ is the ultraviolet radiation received by the earth surface and $U V_{\mathrm{o}}$ is the extraterrestrial ultraviolet radiation. We have been separated these data in optical air mass categories, for each category the mean and standard deviation of the UV radiation were computed. The empirical function describing UV is obtained by a weighted fit of this mean values as shown in Figure 4. 


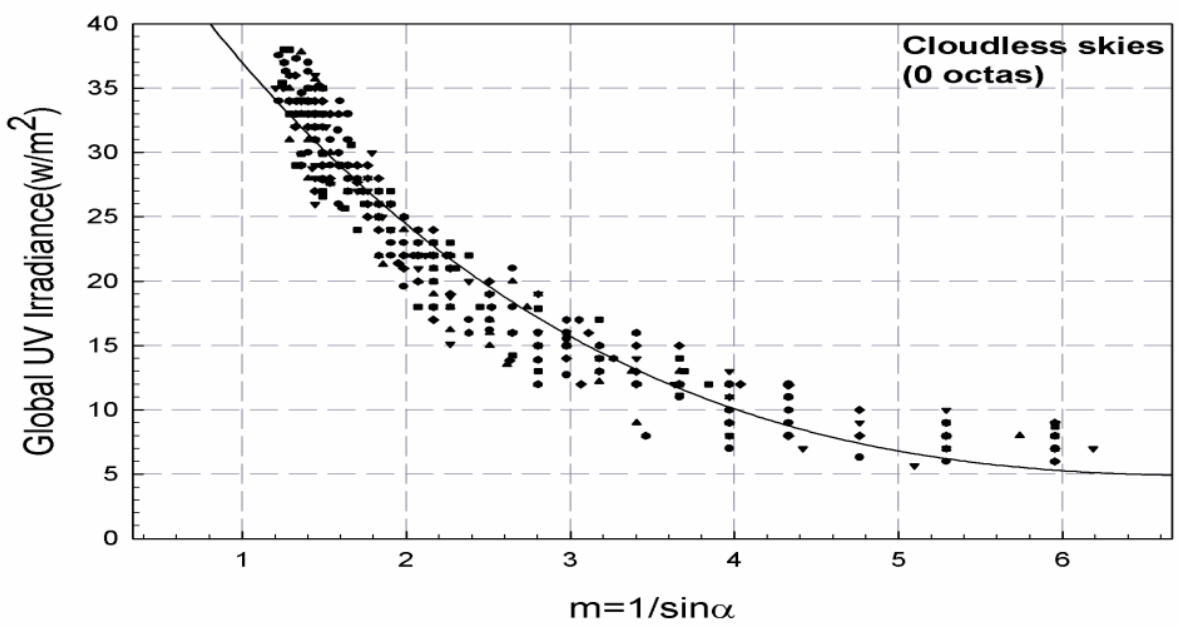

Figure 4. UV global irradiance vs optical air mass.

This $\mathrm{UV}_{0}$ functions has been used to obtain the corresponding $\mathrm{F}_{\mathrm{UV}}$ index that can be considered as a cloud modification factor. Figure (5) presents the performance of this global UV cloud modification factor, as a function of cloud coverage for different sun elevation angle ranges considered in present work.

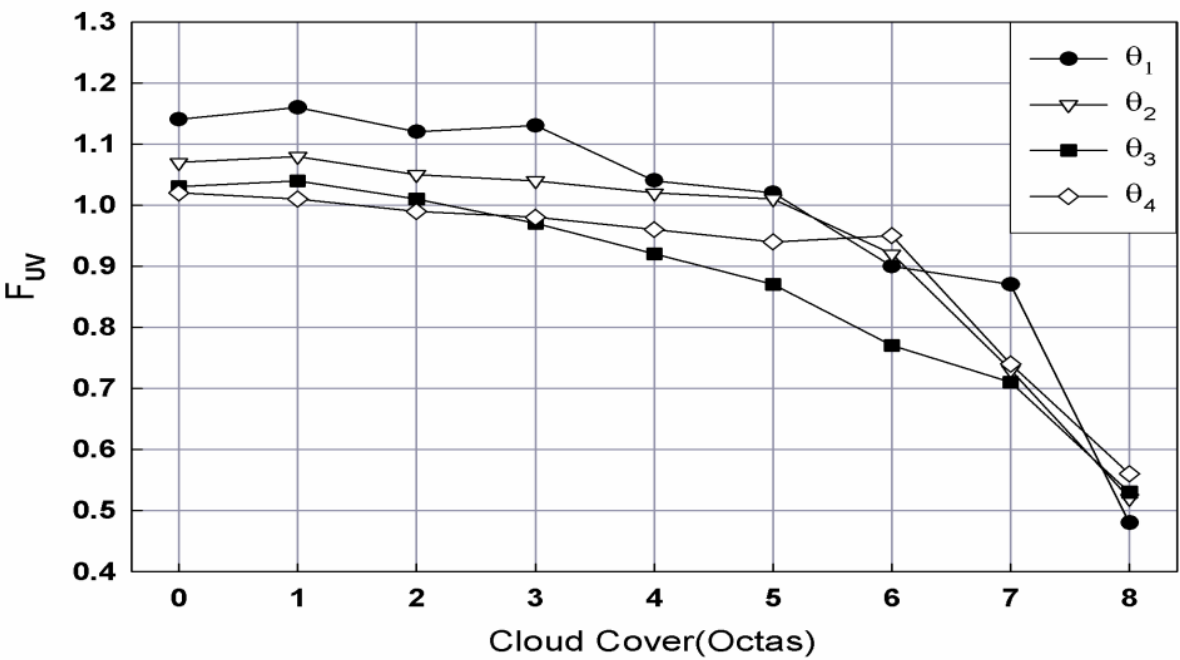

Figure 5. Ultraviolet cloud modification factor $\left(\mathrm{F}_{\mathrm{UV}}\right)$ vs cloud cover for different solar elevation angles.

It can be seen that the behavior of $\mathrm{F}_{\mathrm{UV}}$ is similar for the different ranges of solar elevations. This can be explained as a result of the reflection enhancement under partial cloud cover conditions when geometric conditions are most favorable for the reflection of UV radiation from cloud edges or between base of cloud and the ground surface. For partial cloud cover, this factor presents greater values when solar elevation is high. Under over cast conditions the results obtained for different sun elevation angle ranges $(0.48-0.56)$ according to solar elevations. Grant and Hersler [21] analyzed the UVB irradiance under variable cloud conditions and found a similar trend with an overcast of 0.65 , on other hand, Seckmeyer et al [13] obtained a cloud modification factor that varies between 0.45 in the UVA range and 0.6 in the UVB range.

This difference can be explained by the wavelength dependence of radiation scattering with the cloud, by redistribution of UV radiation due to the cloud.

The standard deviations of the cloud modification factor in Table (1), $\mathrm{F}_{\mathrm{UV}}$, increase with increasing of cloudiness, possibly as a result of variation in cloud opacity. In fact cloud cover alone does not contain explicit information on the optical transmission of clouds. When the sky is only partly cloudily the irradiance values will vary depending on whether the sun in the clear or cloudily portion of sky domes. The nonlinear dependence of $\mathrm{F}_{\mathrm{UV}}$ with cloudiness is largely due to the relatively greater value for intermediate cloudiness. This can be due to reflection from the side of clouds that locally enhanced the radiation levels.

As cloud cover increase beyond 4 or 5 octos, there is a greater chance that the solar disk will be obscured by clouds and thus the attenuation of UV irradiance increase. 
Inspection of Figure (3) and Figure (5) reveals the similarity in the dependence of $\mathrm{K}_{\mathrm{tuv}}$ and $\mathrm{F}_{\mathrm{UV}}$ on cloud cover. This shows the predominance of the cloud effect on UV radiation, evaluated through, $\mathrm{F}_{\mathrm{UV}}$, over the whole atmospheric effect, evaluated by means of Ktuv.

It is possible to compute a cloud modification factor for total solar irradiance,

$$
F_{G}=\frac{G}{G_{O}}
$$

Table 1. Statistical parameters for cloud modification factor for different cloud covers.

\begin{tabular}{|c|c|c|c|c|c|c|c|c|}
\hline \multirow{2}{*}{$\begin{array}{l}\text { Cloud } \\
\text { Cover }\end{array}$} & \multicolumn{2}{|c|}{$\boldsymbol{F}_{U V}$} & \multicolumn{2}{|c|}{$\boldsymbol{F}_{U V}$} & \multicolumn{2}{|c|}{$\boldsymbol{F}_{U V}$} & \multicolumn{2}{|c|}{$\boldsymbol{F}_{U V}$} \\
\hline & $\begin{array}{c}\text { mean } \\
\theta_{1}\end{array}$ & $\begin{array}{l}\mathrm{sd} \\
\theta_{1}\end{array}$ & $\begin{array}{c}\text { mean } \\
\theta_{2}\end{array}$ & $\begin{array}{l}\mathrm{sd} \\
\theta_{2}\end{array}$ & $\begin{array}{c}\text { mean } \\
\theta_{3}\end{array}$ & $\begin{array}{l}\text { sd } \\
\theta_{3}\end{array}$ & $\begin{array}{c}\text { mean } \\
\theta_{4}\end{array}$ & $\begin{array}{l}\mathrm{sd} \\
\theta_{4}\end{array}$ \\
\hline 0 & 1.14 & 0.086 & 1.07 & 0.081 & 1.03 & 0.089 & 1.02 & 0.11 \\
\hline 1 & 1.16 & 0.067 & 1.08 & 0.064 & 1.04 & 0.086 & 1.01 & 0.9 \\
\hline 2 & 1.12 & 0.072 & 1.05 & 0.091 & 1.01 & 0.120 & 0.99 & 0.12 \\
\hline 3 & 1.13 & 0.088 & 1.04 & 0.013 & 0.97 & 0.130 & 0.98 & 0.132 \\
\hline 4 & 1.04 & 0.190 & 1.02 & 0.132 & 0.92 & 0.131 & 0.96 & 0.135 \\
\hline 5 & 1.02 & 0.166 & 1.01 & 0.128 & 0.87 & 0.160 & 0.94 & 0.141 \\
\hline 6 & 0.9 & 0.163 & 0.92 & 0.215 & 0.77 & 0.196 & 0.96 & 0.143 \\
\hline 7 & 0.87 & 0.189 & 0.73 & 0.221 & 0.71 & 0.218 & 0.74 & 0.223 \\
\hline 8 & 0.48 & 0.280 & 0.52 & 0.219 & 0.53 & 0.230 & 0.65 & 0.22 \\
\hline
\end{tabular}

$F_{U V}$ mean: mean values of $F_{U V}, F_{U V}$ sd: corresponding standard deviations.

In order to study the different influences by clouds on both radiative fluxes in figure (6) we show the relation between dimensionless factors ( $F_{U V}=\frac{U V}{U V_{O}}$ and $F_{G}=\frac{G}{G_{O}}$ ). A procedure similar to that followed in the evaluation of $\mathrm{UV}_{\mathrm{o}}$ has been considered in the estimation of $\mathrm{G}_{0}$. After an extensive examination of various curves fitting option, the following functional dependence was found to be the most appropriate:

$$
F_{U V}=-0.016+1.106 F_{G}-0.079 F_{G}{ }^{2}-0.05 F_{G}{ }^{3}
$$

For the above relation $(\mathrm{R}=0.93$ with standard error of estimate $=0.09)$. There is evident departure from the line 1:1 that represent coincident factors, i.e., the clouds does not transmit equally the UV global solar irradiance.

Figure (6) shows that the total global irradiance suffers a proportionally reduction than the UV global irradiance,

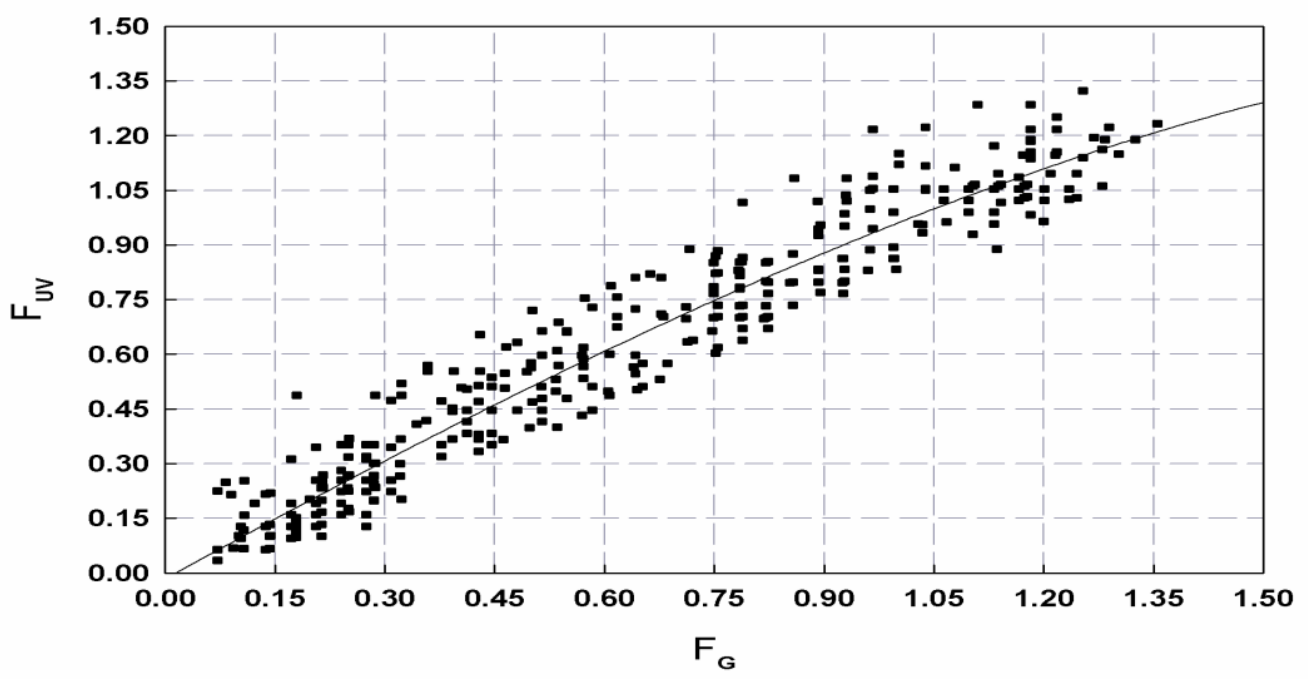

Figure(6): Ultraviolet cloud modification factor $\left(\mathrm{F}_{\mathrm{UV}}\right)$, vs total Global cloud modification factor $\mathrm{F}_{\mathrm{G}}$. 


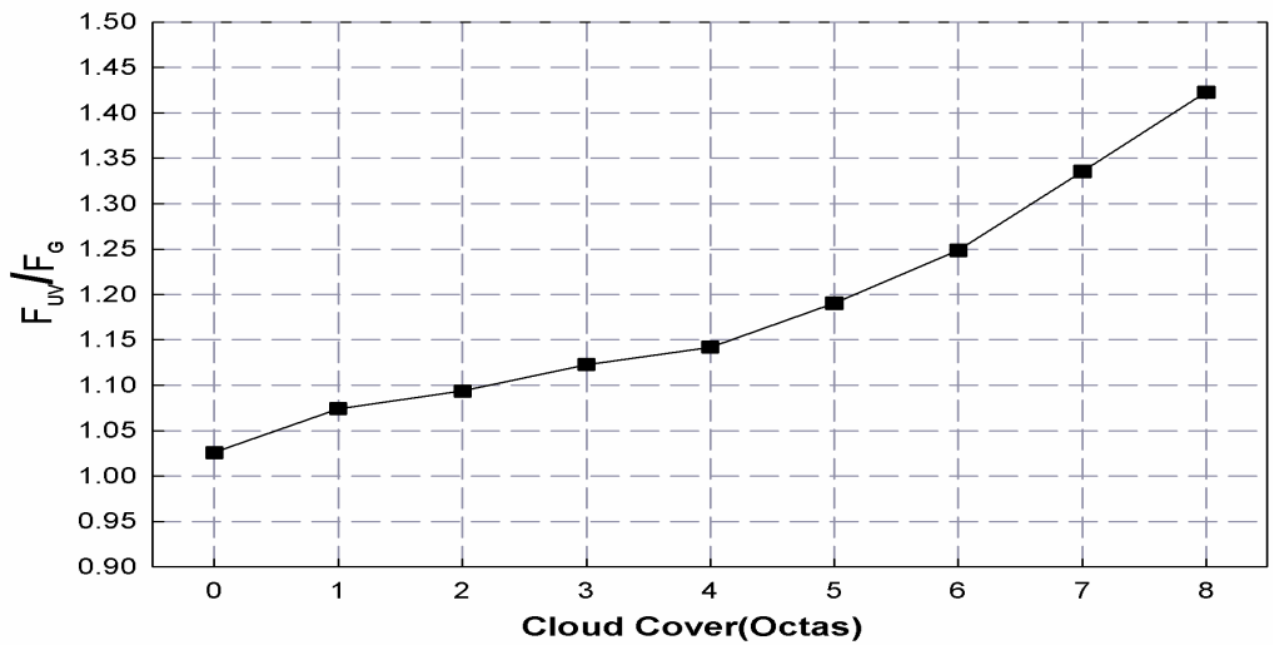

Figure 7. Ratio of UV global cloud modification factor FUV to total global cloud modification factor FG vs cloud cover.

Figure (7) shows the relation between the Ultraviolet cloud modification factor $\mathrm{F}_{\mathrm{UV}}$ and the global cloud modification factor $F_{G}$ as a function of cloud cover. In accordance with figure (2), clouds do not decrease UV radiation as much global radiation.

\section{CONCLUSION}

The influence of the clouds The UV irradiance which measured under partly cloudy and overcast skies was examined and evaluated. The UV irradiance for cloudy skies did not decrease with increasing cloud cover until the cloud cover was greater than 4 octas especially with sun elevation angles larger than $40^{\circ}$. In general, the cloud attenuate the UV irradiance by $(35-52 \%)$ form its total value. Ultraviolet cloud modification factor $\left(\mathrm{F}_{\mathrm{UV}}\right)$ and Global cloud modification factor $\left(\mathrm{F}_{\mathrm{G}}\right)$ were related by polynomial equation.

\section{REFERENCES}

[1] AL-Salihi, A.M. 2008' Effect of Some Atmospheric Factors on Ultraviolet Radiation ' Ph.D. thesis, Department of Atmospheric sciences, College of Sciences, Al-Mustansiriyah University.

[2] Al-Aruri, S.D., Rasas, M., Al-Jamal, K. and Shaban, N. 1988. 'An assessment of global ultraviolet solar radiation in the range (0.290-0.385) $\mathrm{mm}$ in Kuwait', Solar Energy, 41, 159- 164.

[3] Al-Aruri, S.D. 1990. 'The empirical relationship between global radiation and global ultraviolet (0.290-0.385) mm solar radiation components', Solar Energy, 45, $61-64$

[4] Elhadidy, M.A., Abdel Nabi, D.Y. and Kruss, P.D. 1990. 'Ultraviolet radiation at Dhahran, Saudi Arabia', Solar Energy, $44,315-319$.

[5] Feister, U. and Grasnick, K.H. 1992. 'Solar UV radiation measurements at Postdam (52² ${ }^{\circ} \mathrm{N}, 13^{\circ} 5^{`} \mathrm{E}$ )', Solar Energy, 49, $541-$ 548.

[6] Khogali, A. and Al-Bar, O.F. 1992. 'A Study of solar ultraviolet radiation at Makkah solar station', Solar Energy, 48, 79 87. Lenoble, J. 1993. Atmospheric Radiati6e Transfer, Deepak Publishing, Hamptom, VA.

[7] Mart · inez-Lozano, J.A., Casanovas, A.J., Utrillas, M.P., 1994. Comparison of global ultraviolet (295385 nm) and global irradiation measured during the warm season in Valencia, Spain. Int. J. Climatol. 14, 93-102.

[8] Fro" hlich, C. and London (eds.). 1986. Raised Instruction Manual on Radiation Instruments and Measurements, WCRP Pub. Series No 7, WMO/TD No 149, pp. 140.

[9] Horneck, G. 1995. 'Quantification of the biological effectiveness of environmental UV radiation', J. Photochem. Photobiol. B: Biology, 31, $43-49$.

[10] Lorento. J., Redano. A., Decabo, x., 1996. Influence of urban aerosol on spectral solar irradiance. Appl. Meteorl, 33, 406-415.

[11] Diaz, j. p., Exposito, F.J., Torres., C.J. Herra., 2001. Radiative properties of aerosols in Saharan dust out breaks using ground based and satellite data, J. Geophys. Res. Atmos. 106, 18403-18416.

[12] Wenny, B.N., saxena, V.K., 2001. Aerosol optical depth measurements and their impact on surface level of ultraviolet-B radiation, Geophys.Res.

[13] Seckmeyer, G., Erb, R., Albold, A., 1996. Transmittance of a cloud is wavelength-dependent in the UV-range. Geophys. Res. Lett. 23, 27532755.

[14] Alados, I., Olma, F.J. FOYO - Moreno .I (2000) Estimation of photo synthetically active radiation under cloudily conditions. Agricult. Forest Meteorology. 102,44-50.

[15] Canada, G., Pedros, G., Lopez, A., Bosca, J (2000) Influence of the clearness index for the clearness index for the spectrum and of the relative optical air mass on UV irradiance two locations in the Mediterranean area, Valencia and Cordoba, J. Geophys. 105, 4759-4766.

[16] Foyo-morrno, I., vida., J., Alados-Arboledos (1998) Ground based ultraviolet (295-385 nm) and broad bond solar radiation measurements in south-Eastren Spain. INT. J. climatological, 18, 1389-1400. 
[17] Ambach,W., Blumthalar, M., Wendler, G (1991) Comparison of Ultraviolet radiation at an arctic and an alpine site, solar Energy. 47, 121, - 126 .

[18] Martineg-Lozano, J.A., Casanovas, J., Utrills, M.P (1994). Comparison of global ultraviolet (295-385 nm) and global radiation on measured during warm season in Valencia, spain.INT. J. climatological, 14, 93-102.

[19] Lenoble, J (1993). Atmospheric Radiative transfer, Deepak publishing, Hampton, VA, pp. 523.

[20] Gueymard, C (2005). SMARTS code, version 2.9.5, user manual, solar consulting service, USA.

[21] Grant, R.H., Heisler, G.M (2000) Estimation of ultraviolet- B irradiance under variable cloud conditions. J. Appl. Meteorology, 39, 904-412. 